Int. J. Electrochem. Sci., 13 (2018) 11730 - 11740

International Journal of

ELECTROCHEMICAL

SCIENCE

www.electrochemsci.org

\title{
Low-potential Electrosynthesis and Fluorescence Properties of Poly(9,9'-bifluorenylidene) in Boron Trifluoride Diethyl Etherate
}

\author{
Nannan Jian ${ }^{1}$, Wenna Zhang ${ }^{2}$, Kai $Q u^{1}$, Shuai Chen ${ }^{2}$, Baoyang Lu ${ }^{2, *}$, Ximei Liu ${ }^{2, *}$, Jingkun Xu ${ }^{1,3, *}$ \\ ${ }^{1}$ School of Chemistry \& Chemical Engineering, Jiangxi Science \& Technology Normal University, \\ Nanchang 330013, Jiangxi, China \\ ${ }^{2}$ School of Pharmacy, Jiangxi Science \& Technology Normal University, Nanchang 330013, Jiangxi, \\ China \\ ${ }^{3}$ School of Chemistry and Molecular Engineering, Qingdao University of Science and Technology, \\ Qingdao 266042, Shandong, China \\ *E-mail: 1by1258@163.com and xujingkun1971@yeah.net
}

doi: $10.20964 / 2018.12 .76$

Received: 14 August 2018 / Accepted: 12 October 2018 / Published: 5 November 2018

Electrosynthesized polyfluorene-based conjugated polymers (PFs) have been extensively researched since their discovery owing to their excellent fluorescence properties and one step fabrication process. However, most polyfluorenes have been obtained under high polymerization potentials over $1.2 \mathrm{~V}$ vs. $\mathrm{Ag} / \mathrm{AgCl}$, resulting in inevitable overoxidation and structural defects of the deposited polymer films, and thus imposing instability and long-term deterioration in optoelectronic properties. Herein, by the employment of a fused 9,9'-bifluorenylidene (BFY) as the initial monomer and boron trifluoride diethyl etherate (BFEE) as both the solvent and supporting electrolyte for the electropolymerization, we successfully achieved the low potential electrodeposition of a novel polyfluorene film for the first time at $0.9 \mathrm{~V}$ vs. $\mathrm{Ag} / \mathrm{AgCl}$. We demonstrate that the polymerization mechanism of $\mathrm{BFY}$ occurs dominantly at the 2,7 positions of the fluorene unit and forms an interconnected network of polyfluorene segments. The electropolymerization of BFY displays a sphere-type deposition process to produce a surface morphology of nanosphere clusters. We further explore the thermal degradation and electronic property evolution from monomer BFY to the deposited polymer, and find that the polymer exhibits a $100 \mathrm{~nm}$-red shift in electronic absorption and significantly enhanced fluorescence intensity with a high quantum yield up to 0.78 .

Keywords: conjugated polymers, electropolymerization, 9,9'-bifluorenylidene, boron trifluoride diethyl etherate, fluorescence

\section{$\underline{\text { FULL TEXT }}$}


(C) 2018 The Authors. Published by ESG (www.electrochemsci.org). This article is an open access article distributed under the terms and conditions of the Creative Commons Attribution license (http://creativecommons.org/licenses/by/4.0/). 